\title{
Effects of grazing by herbivorous gastropod (Trochus niloticus) on the survivorship of cultured coral spat
}

\author{
Ronald D Villanueva ${ }^{1 *}$, Maria Vanessa B Baria ${ }^{1,2}$ and Dexter W dela Cruz ${ }^{1,3}$
}

\begin{abstract}
Background: The technology in the production of sexual coral propagules for coral reef restoration is being actively developed recently to address concerns on the low genetic diversity in coral populations restored through transplantation of coral materials derived from fragmentation.

Results: In this study, we produced coral spat attached to rubble and determined the effect of the addition of hatchery-reared trochus, a herbivorous gastropod, at two densities (one and two trochus per cage, equivalent to four and eight trochus per square meter, respectively) on the survivorship of the spat cultured in cages at the hatchery and in situ nursery. After 5 weeks of culture in the hatchery, spat survivorship was significantly higher in the two per cage trochus treatment $(18.3 \% \pm 6.7 \%$, mean \pm sd) than in the control $(5.5 \% \pm 1.7 \%)$, with the one per cage trochus treatment having intermediate spat survival (12.6\% $\pm 7.9 \%)$. The enhanced spat survivorship, though not apparent in the in situ nursery culture, is attributed to the grazing effect of trochus as significantly lower turf algal cover was observed in rubble placed in two per cage trochus treatment $(44.8 \% \pm 3.2 \%)$ than in the one per cage trochus treatment and control $(80.2 \% \pm 6.5 \%$ and $76.3 \% \pm 5.0 \%$, respectively).
\end{abstract}

Conclusions: This study demonstrates that trochus can be used to control algal cover and enhance survivorship of coral spat in caged culture, at least in the hatchery, for the production of sexually derived transplant materials for coral reef restoration.

Keywords: Coral; Restoration; Trochus niloticus; Acropora valida

\section{Background}

Coral reefs worldwide are experiencing unprecedented decline from anthropogenic and natural stressors (Wilkinson 2004; Burke et al. 2011). Aside from protecting the remaining coral reefs, degraded ones should be actively restored and properly managed. Several coral reef restoration tools were put forward during the past three decades, with coral transplantation on the forefront (Rinkevich 2005). Recent advances on coral transplantation involved the use of nursery-reared corals, i.e., based on the 'coral gardening' concept (sensu Rinkevich 1995, 2000, 2006; Epstein et al. 2001, 2003). Coral gardening would enable the production of large quantities of

\footnotetext{
* Correspondence: villanuevr@yahoo.com

${ }^{1}$ The Marine Science Institute, College of Science, University of the Philippines Diliman, Quezon City 1101, Philippines

Full list of author information is available at the end of the article
}

transplant materials, thereby minimizing collateral damage to source reefs (Edwards and Gomez 2007).

In the production of sexually derived transplant materials for reef restoration, herbivorous topshell, Trochus niloticus, was co-cultured with coral juveniles in cages deployed in situ (Omori 2005). Although success of the mass culture of sexually derived transplant materials was attributed to algal control by trochus (Omori 2005), the effects of trochus on algal growth and juvenile coral survivorship were not systematically demonstrated.

The early after-settlement or early juvenile stage is when the highest mortality rates are experienced by corals (Babcock 1985), as by other benthic marine invertebrates (Gosselin and Qian 1997). Therefore, improvement in culture conditions during this life stage can potentially result to a remarkable increase in the production of coral transplant materials for reef restoration.

\section{实}


The present study aims to determine the effects of trochus on the survivorship and growth of coral spat (early juvenile stage) cultured in the hatchery and in situ nursery.

\section{Methods}

\section{Collection and transportation of coral}

Twenty mature colonies of Acropora valida were collected from the reef beside Cory sandbar at the BolinaoAnda Reef Complex (BARC), Pangasinan, northwestern Philippines $\left(16^{\circ} 19^{\prime} 36^{\prime \prime} \mathrm{N}, 120^{\circ} 02^{\prime} 01^{\prime \prime}\right.$ E) in June 2010 at around full moon. Collected mature colonies were transported to the hatchery facility of the Bolinao Marine Laboratory of The Marine Science Institute (University of the Philippines) for ex situ observation of spawning and gamete collection.

\section{Spawning and fertilization}

Colonies were held in flow-through cement tanks $(0.7-\mathrm{m}$ water depth, $3.5-\mathrm{m}^{3}$ volume) with aeration. During the succeeding nights, spawning was checked by periodic monitoring of colonies from 1800 to 2200 hours. Seawater flow and aeration were cut off during each monitoring period and turned back on thereafter. All colonies were held in the same tank for spawning. Spawning occurred 5 to 7 days after the full moon (June 30 to July 2, 2010) between 1930 and 2030 hours, with at least seven colonies spawning each night. Gamete fertilization, larval culture, and settlement competency methods followed Heyward and Negri (1999) and Baria et al. (2010) with minor modifications - including the use of untreated seawater (no employment of UV sterilization and micron-size filtration), shortened fertilization time (30 min), and employment of the second sperm wash.

\section{Developing embryos and larval settlement}

Developing embryos were then transferred to cylindrical tanks (water surface area $1.1 \mathrm{~m}^{2}$ ) with approximately 1,500 1 of seawater at densities between 250 and $300 \mathrm{l}^{-1}$ and allowed to stand for $12 \mathrm{~h}$ without aeration. After which, half of the culture seawater was changed, and subsequent changes were performed on a daily basis. Aeration was supplied only after $24 \mathrm{~h}$ from fertilization, as developing larvae at earlier stages (several hours postfertilization) are delicate and can be broken by aerationgenerated turbulence.

Larval settlement competency was determined daily from 2 days post-fertilization. To induce settlement, a coral rubble chip (approximately $0.5 \mathrm{~cm}^{2}$ ) with crustose coralline algae (CCA) was added to ten larvae in $15 \mathrm{ml}$ of seawater contained in polystyrene culture wells $(n=6)$. Competency was examined after $24 \mathrm{~h}$ with the aid of a stereomicroscope, as indicated by the transformation of a larva to a flattened, disc-shaped mass which is attached to the rubble or wall of the culture well. Settlement peaked 8 days post-fertilization (settlement $73 \% \pm 33 \%$, mean $\pm \mathrm{sd}$ ).

Coral rubble with patches of CCA was used as settlement substrate for the A. valida larvae. Rubble pieces (10- to $16-\mathrm{cm}$ length and 1 - to $1.5-\mathrm{cm}$ diameter) were dead fragments of staghorn Acropora spp. and were collected in the rubble deposition zone at the back reef near Silaqui Island at BARC $\left(16^{\circ} 26^{\prime} 32^{\prime \prime} \mathrm{N}, 119^{\circ} 54^{\prime} 36^{\prime \prime} \mathrm{E}\right)$. Any macroinvertebrate and turf or fleshy macroalga attached to the rubble pieces were removed by hand or with the use of soft-bristled brush prior to use. Rubble pieces were offered to coral larvae 7 days post-fertilization, i.e., when settlement competency is about to peak. Rubble pieces were submerged to larval suspension (150 to 200 larva $\left.~^{-1}\right)$ in a settlement bin $(50 \times 40 \times 30 \mathrm{~cm}, l \times w \times h$, filled up to $50 \mathrm{l}$ ), supplied with mild aeration. Larvae were allowed to settle and metamorphose within 4 days, after which flow-through seawater was supplied. Newly settled coral spat were reared in this system in the hatchery for approximately 2 weeks in order for ample calcification to occur (full development of primary polyp corallum which is approximately 7 days after settlement in Acropora millepora, Babcock et al. 2003). Rubble with coral spat was carefully cleaned with soft-bristled brush to remove microalgal mat and non-coralline macroalgae prior to use in the caging experiments. Coral spat density in rubble and spat diameter were determined under a stereomicroscope to be $0.5 \pm 0.2 \mathrm{~cm}^{-2}(n=18)$ and $0.96 \pm 0.53 \mathrm{~mm}$ $(n=547)$, respectively.

\section{Effects of trochus addition on spat survivorship in the hatchery}

The grazing gastropod $T$. niloticus was reared in the hatchery as described in Villanueva et al. (2010). Individuals used in the following experiments were sub-adults with greatest basal diameter of $26 \pm 2 \mathrm{~mm}(n=12)$.

To determine the effects of trochus addition on spat survivorship, caging experiments were conducted. Cages used were made up of plastic trays $(33 \times 22 \times 10 \mathrm{~cm}, l \times$ $w \times h$; with $0.3 \times 0.3 \mathrm{~cm}$ openings on the sides and $0.3 \times$ $0.1 \mathrm{~cm}$ openings on the bottom) covered with polyethylene screen (Amazon $^{\mathrm{nM}}$ net, Valenzuela City, Philippines; $1 \times 1 \mathrm{~cm}$ openings, top portion of the cages). Three randomly selected rubble pieces, with $11 \pm 4$ spat per rubble piece, were placed inside each of 18 cages. Each rubble was secured inside the cage (lower portion) using a cable tie. The exact number of spat in each cage (experimental unit) was counted, with each receiving $32 \pm 12$ spat. There were three trochus treatments (viz. control, one per cage, and two per cage) deployed in each of two culture treatments (hatchery and in situ nursery), with three replicate cages per treatment combination. One trochus per cage corresponds to four trochus per square meter, while two trochus per cage corresponds to eight 
trochus per square meter. These density levels were considerably higher than the averaged densities of trochus in the wild (approximately one individual $\mathrm{m}^{-2}$ (Secretariat of the Pacific Community 2008)) in order to test if enhanced grazing has an effect on cultured spat survivorship and growth. There were no significant differences in the shell diameter of trochus allocated among the treatments (two-way analysis of variance (ANOVA); culture: $F=4.84$, $p=0.06$; trochus density: $F=0.21, p=0.66)$. Though the difference in the diameter of trochus used between the two culture treatments is just a little above the arbitrary statistical threshold (which is still acceptable to be obtained from a homogeneous population), all the trochus used were at the sub-adult stage and are of the same cohort (same age), as well as cultured together at the hatchery.

The hatchery (ex situ) culture system was a concrete tank (0.7-m water depth, $3.5-\mathrm{m}^{3}$ volume) with flowthrough seawater (approximately $20 \mathrm{l} \mathrm{min}^{-1}$ ) and aeration. Experimental cages were placed on the bottom of three tanks, with each tank receiving a replicate cage of each trochus treatment level. The three tanks were approximately $1 \mathrm{~m}$ apart. The cages in a tank were randomly interspersed, with approximately $30-\mathrm{cm}$ distance between cages.

\section{Effects of trochus addition on spat survivorship in the in situ nursery}

The in situ nursery was elevated from the substrate by $1 \mathrm{~m}$ using angle bars driven to the substrate serving as stands. Another set of experimental cages (similar to those in the hatchery) attached using cable ties to parallel angle bars (6 m long, $30 \mathrm{~cm}$ apart) were assembled and secured on top of the angle bar stands. The cages were clustered into three, with each cluster composed of a replicate of each trochus treatment level. Cages in each cluster were approximately $30 \mathrm{~cm}$ apart, and there is a distance of approximately $1 \mathrm{~m}$ per cluster. The treatment levels in each cluster were randomly interspersed. The nursery was located on the lagoon near Binabalian Labas at BARC $\left(16^{\circ} 25^{\prime} 37^{\prime \prime} \mathrm{N}, 119^{\circ} 54^{\prime} 52^{\prime \prime} \mathrm{E}\right)$.

\section{Environmental monitoring, maintenance of cage, and microscopic examination}

Environmental parameters (salinity, water motion, light attenuation, and sedimentation rate) in both culture setups were monitored once, at the start of the caging experiment, following the techniques described in Villanueva et al. (2005).

The caging experiment ran for 36 days in August to September 2010, without any maintenance procedure. This study period (initially thought to be 1 month but extended for several days due to diving constraints to retrieve the cages in the in situ nursery) was selected to study the effects of macroalgae and trochus on the early post-settlement stage of coral juveniles. During retrieval, pieces of rubble from each cage were thoroughly searched for live Acropora spat under a stereoscope. Throughout the course of the caging experiment, no acroporid coral is predicted to spawn in the area (peak of spawning at the study site occurs during March to May (Vicentuan et al. 2008)); hence, no Acropora larvae from the wild were expected to have settled into the experimental rubble. A. valida is one of the last Acropora species to spawn during the reproductive period in BARC. Our observation at the hatchery indicates that if the larvae were offered (at 7 to 8 days post-fertilization) with rubble laden with CCA or any substrate conditioned in seawater for several weeks, most of these $(>80 \%)$ settle and metamorphose (see also Heyward and Negri 1999). The observation of Graham et al. (2008) on high longevity, i.e., long planktonic (larval) duration, was for larvae that were maintained in $0.2-\mu \mathrm{m}$ filtered (sterile) seawater and in clean culture vessels (with periodic transfer) at the laboratory. In the absence of settlement cues, the larvae in this condition are expected to remain in the water column for prolonged periods. At natural conditions (in the reef), aside from the overwhelming presence of settlement cues, larvae can be easily depleted through predation or transport off-reef. Hence, overestimation of coral juveniles on the rubble at the in situ nursery is not likely.

After microscopic examination for live acroporid spat, a randomly selected portion (approximately $2 \mathrm{~cm}^{2}$ ) in the rubble was digitally photographed under the stereoscope. Ten random points were generated in each of the rubble photograph using Coral Point Count with Excel extensions software (Kohler and Gill 2006). The benthic composition under each point was classified either as bare, sponge, or one of the following algal functional groups: turf/filamentous, crustose coralline, corticated, and foliose. Different algal functional groups on coral substrates were differentiated since they exhibit variable effects to coral survival and growth (McCook et al. 2001). Since crustose coralline algae in experimental rubble pieces were not removed prior to the experiment, this benthic category was not included in the data analysis.

The greatest diameters of coral spat were determined using an ocular micrometer under a stereoscope prior to the caging experiment. Diameters of all surviving spat after the experiment were also measured per treatment. As coral spat were not individually tagged and considerable mortality was incurred after the experiment, spat growth rates were not calculated. For the co-cultured trochus, on the other hand, no mortality was observed; hence, trochus growth rate based on initial and final greatest basal diameter (measured using a caliper) was determined. 


\section{Data analyses}

One-way ANOVA was used to determine the effects of trochus (control, one per cage, and two per cage) on survivorship of coral spat cultured in the hatchery and in situ nursery. Furthermore, to determine the interactive effects of trochus and culture (hatchery and in situ nursery) treatments on spat survivorship, a two-way ANOVA was performed. Survivorship data met the parametric assumptions of normality (Ryan-Joiner test) and equal variances (Bartlett's and Levene's tests). These parametric procedures were performed with Minitab ${ }^{\oplus}$ statistical software. Two-way ANOVA was also performed to detect differences in the diameter of surviving spat among trochus and culture treatments.

Differences in cover of turf/filamentous algae (the major functional group of algae recorded in the rubble substrates, comprising $88 \%$ to $100 \%$ of benthic life cover excluding CCA) among treatments (trochus and culture) were determined by two-way ANOVA, with subsequent post hoc comparison with Tukey's test. Turf cover data were Johnson-transformed to meet the parametric assumptions. Differences in growth rates of trochus between the trochus density and culture treatments were also determined by two-way ANOVA. For minor components of the benthic life in the coral rubble (sponge, foliose algae, and corticated algae), differences in cover among trochus treatments in each culture setup were examined using the non-parametric Kruskal-Wallis test. Due to the presence of many zero values for these components, the dataset did not satisfy the parametric assumptions.

\section{Results}

The environmental parameters were significantly different between the two culture setups, with sedimentation rate, water motion, and light attenuation significantly higher in the in situ nursery than in the land-based hatchery (Table 1). Salinity was similar in these two setups.

No trochus died in the cages within the 36-day experiment both in the hatchery and in situ nursery. Minimal growth was recorded for trochus in the cages maintained in the hatchery, while relatively higher growth rates were obtained for trochus in both trochus density treatments deployed in the in situ nursery (Figure 1). Trochus growth was significantly affected by culture setup
(ANOVA, $F=19.28, p=0.002$ ), but not by trochus density $(F=2.37, p=0.162)$. No interactive effect between the two factors was detected $(F=2.14, p=0.181)$.

The survival of acroporid spat in the two trochus densities and the control is similar in cages held at the in situ nursery for 36 days (approximately 16\% to $18 \%$, Figure 2a). At the hatchery, significantly higher spat survivorship was recorded in the two per cage treatment compared to the control (Figure 2a, $F=10.39, p=0.032$ ). Spat survivorship for one per cage trochus treatment, on the other hand, was not significantly different to the control and two per cage treatment (Figure 2a, $F=2.34$ and $0.94, p=0.200$ and 0.388 , respectively). In general, the main and interactive effects of trochus and culture treatments on spat survivorship were not significant (trochus: $F=0.88, p=0.439$; culture: $F=1.34, p=0.270$; trochus $\times$ culture: $F=0.59, p=0.570)$.

Sizes of surviving coral spat after the experimental culture (Figure $2 \mathrm{~b}$ ) did not differ significantly among trochus $(F=0.13, p=0.878)$ and between culture $(F=0.002$, $p=0.968)$ treatments.

Turf/filamentous algae comprised $45 \%$ to $90 \%$ of the substrate cover or $88 \%$ to $100 \%$ of the total benthic live cover in experimental rubble pieces in the different treatments (Figure 3). Both trochus $(F=11.19, p=0.002)$ and culture $(F=9.96, p=0.008)$ treatments, but not their interaction $(F=0.49, p=0.626)$, significantly affected turf algal cover in the rubble. Generally, higher turf algal cover was recorded in the rubble pieces deployed in the in situ nursery than in the hatchery (Tukey's test, $p<0.05$, Figure 2c). In both culture setups, turf algal covers in rubble pieces maintained in the two per cage trochus treatment were significantly lower than those in the one per cage trochus treatment and in the control $(p<0.05$, Figure 2c). Cover for minor benthic components did not differ significantly among trochus treatments in the in situ nursery (Kruskal-Wallis test; sponge: $H=1.80, p=0.407$; foliose algae: $H=2.29, p=0.318$; corticated algae: $H=$ $0.62, p=0.733$ ) and at the hatchery (sponge: $H=0.00, p=$ 1.000; foliose algae: $H=0.60, p=0.741$; corticated algae: $H=2.40, p=0.301$ ).

\section{Discussion}

Herbivorous macroinvertebrates are significant grazers in some reef habitats (Klumpp and Pulfrich 1989). The enhancement of grazing gastropod (trochus) populations

Table 1 Comparison of environmental parameters (mean $\pm s d, n=3$ ) at the two setups

\begin{tabular}{|c|c|c|c|c|}
\hline Parameter & Hatchery & In situ nursery & $F$ & $p$ value \\
\hline Sediment accumulation rate $\left(\mathrm{g} \mathrm{m}^{-2}\right.$ day $\left.^{-1}\right)$ & $4.4 \pm 2.0$ & $104.8 \pm 53.8$ & 10.43 & 0.032 \\
\hline Water motion (diffusion index) & $0.92 \pm 0.14$ & $1.88 \pm 0.08$ & 105.40 & 0.001 \\
\hline Light attenuation (\%) & $66.6 \pm 0.8$ & $87.9 \pm 0.6$ & $1,215.62$ & $<0.001$ \\
\hline Salinity $(\%)$ & $34 \pm 0$ & $34 \pm 0$ & 0 & 1.0 \\
\hline
\end{tabular}



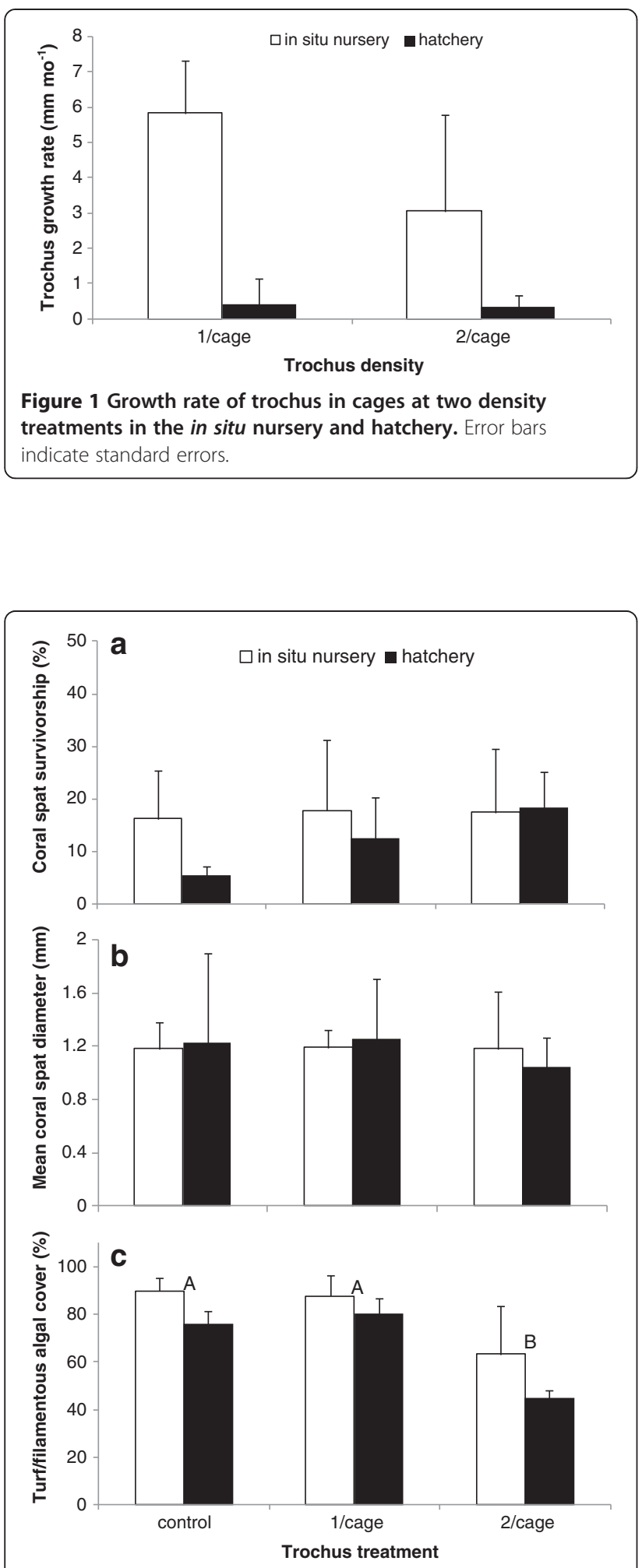

Figure 2 Coral spat survivorship (a), diameter of surviving spat (b), and turf/filamentous algal cover (c). Spat in rubble pieces were maintained in different treatments for 36 days. Trochus treatment levels with similar uppercase letters above bars in panel $c$ are not significantly different (Tukey's test, $p>0.05$ ). Error bars indicate standard deviations.

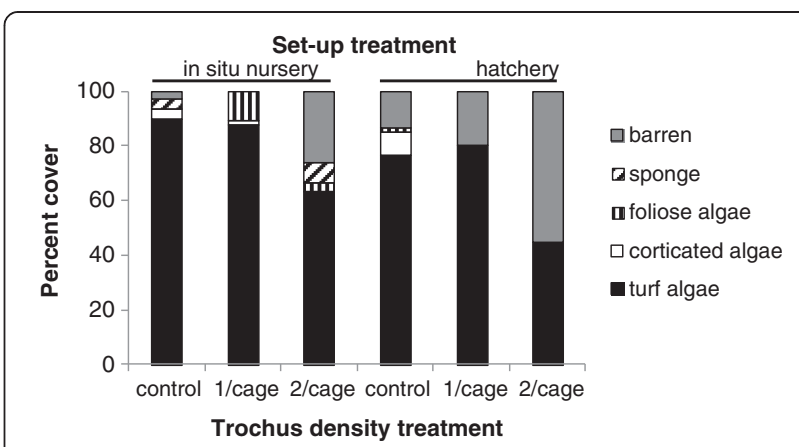

Figure 3 Benthic composition of the surface of experimental rubble containing coral spat subjected to different treatments.

has the potential of enhancing the recovery of degraded reef areas through algal grazing and subsequent increase in coral recruitment rate, though technical hurdles in this restoration approach, e.g., predation on restocked individuals, have yet to be addressed (Villanueva et al. 2010). In trochus stock enhancement experiments which were not specifically for coral reef restoration, low survival rates were also recorded and improved approaches in restocking have been suggested (Crowe et al. 2002; Purcell and Cheng 2010). Rapid recruitment of corals on concrete structures to culture trochus has been documented and hypothesized to be due to the grazing effect of trochus creating suitable substrate for coral recruitment (Omori et al. 2006).

In conditions where predators are excluded, e.g., in cages, trochus can be utilized to reduce algal biomass and enhance survivorship and growth of organisms cocultured with trochus. This has been previously demonstrated in caged giant clam-trochus co-culture for the production of the former to restock coral reefs with depleted populations (Clarke et al. 2003). In the present study, trochus under caged conditions produced a change in benthic composition in substrates containing coral spat primarily due to decrease in the turf/filamentous algal cover and subsequent increase in the barren cover. However, this effect is only apparent at the two per cage trochus treatment. Interestingly, the effect cascades to enhancement of coral spat survivorship in the hatchery culture. This result lends support to the earlier account on the success of mass culture of Acropora corals from spat, with trochus for algal control (Omori 2005). Turf algae have been established to be detrimental to spat survivorship (reviewed in Birrell et al. 2008).

Though the algal diet preference of T. niloticus is not within the scope of this study, it is worthy to mention that in the congeneric Trochus maculatus, filamentous/ turf-forming algae is preferred over corticated and foliose algae ( $\mathrm{Ng}$ et al. 2013). This previous observation is consistent with the findings of the present study as the 
cover of turf, but not corticated and foliose, algae was significantly lower in rubble pieces exposed to trochus (at two per cage density) than that in the control. In both culture systems (hatchery and in situ nursery), turf algae covered most of the rubble substrate in the control cages. Considering the type of macroalgae expected to foul a particular culture system and incorporating the dietary habit and consumption rate of grazers $(\mathrm{Ng}$ et al. 2013), the effectiveness of biological control of fouling macroalgae in mariculture can be considerably enhanced. Furthermore, it has been suggested that the dietary habit of grazers would have shaped the fouling assemblage in mariculture ( $\mathrm{Ng}$ et al. 2013). In the present study, the decrease in turf algal cover due to trochus grazing corresponded to an increase in the cover of barren surfaces but not in the cover of other (non-turf) algal forms. The unavailability of propagules or suboptimal growth of the other algal forms in the culture systems during the experimental period can possibly provide explanation to this observation.

Trochus addition in cages at one trochus per cage did not produce any effect on turf algal cover and spat survivorship, indicating below threshold grazer density for algal cover control. A similar effect was observed in the culture of giant clams with trochus, where a trochus density of five per cage $\left(0.36 \mathrm{~m}^{2}\right.$ with $18-\mathrm{mm}$ mesh, equivalent to $14 \mathrm{~m}^{-2}$ ) did not produce enhancement in clam survivorship and growth, while a higher density (ten per cage, equivalent to $28 \mathrm{~m}^{-2}$ ) did produce such effect (Clarke et al. 2003). A threshold grazer density should, then, be determined when incorporating trochus in caged culture of other reef organisms. This threshold trochus density may be dependent to the nature/type of co-cultured organism and to the culture setup or site. Some organisms may be more sensitive to algal effects and therefore require a higher grazer density. Since there was no observed effect of trochus treatment on spat survivorship in the in situ nursery culture (where environmental parameters were found to be different to that in the hatchery), even at two per cage trochus density, the efficiency of trochus to enhance the survival of coral spat in caged culture is then variable in different culture conditions. For hatchery conditions, the recommended density of trochus to effectively control turf/filamentous algae and thereby enhance spat survivorship is at least eight sub-adult individuals per square meter of cage surface area.

There was a higher spat survivorship for the control in the in situ nursery than in the nursery, indicative of more conducive culture conditions (higher water motion and light intensity, despite higher sedimentation rate) in the former than in the latter. Since there is absence of trochus effect on spat survival in the former and presence of effect in the latter, at least one of these conditions could be counteracting the expected positive effect of trochus at the former culture setup. Trochus addition, at two per cage, resulted to lower turf cover (Figure 2c). As turf algae have been shown to trap sediments (Purcell 2000), a lower sediment load is likely at this trochus treatment level. However, sediment negatively affects coral spat survivorship (e.g., Sato 1985). Further studies should be done to elucidate the mechanism underlying the absence of trochus effect on spat survival despite lowered turf algal cover at the in situ nursery.

Trochus did not eat nor scrape coral polyps (Omori et al. 2007). Although the foot and mouth of trochus are made up of soft tissues, scraping or dislodgement can possibly occur when spat is not yet extensively calcified, i.e., during the first few days post-settlement. This was shown in 3-day Acropora digitifera and 5-day Acropora tenuis spat where significant scraping was observed when co-cultured with trochus (approximately 10-mm mean basal diameter) at $520 \mathrm{~m}^{-2}$ (Tamura 2008). In $A$. millepora recruit, the corallum is fully developed (from basal plate to coenosteum and septa) at 7 days postsettlement (Babcock et al. 2003). The spat of $A$. valida in this study were approximately 2 weeks old at the start of co-culture with trochus. In pocilloporid corals, trochus can be offered earlier to spat culture as skeletogenesis in the primary polyp has been found to be almost complete at 3 days post-settlement (Baird and Babcock 2000). Considering the variability in the duration of the completion of primary polyp skeletogenesis among coral species, future studies should determine the timing of introduction of trochus to spat culture such that dislodgement or accidental grazing can be limited.

\section{Conclusions}

The introduction of grazers has been proven to be effective in controlling fouling macroalgae in the culture of coral spat (Toh et al. 2013; this study). Aside from being effective, such biological control measure is environmentfriendly, contrasting to the use of antifouling chemicals (such as copper-containing paints) that had been earlier suggested in the culture of small coral nubbins (Shafir et al. 2009). Aside from these, the use of trochus can be less costly and less time-consuming as compared to maintenance procedures to manually remove fouling algae in culture systems. Hence, trochus co-culture can be of use in the production of large numbers of sexually derived corals to meet the demand for large-scale reef restoration through enhancement of coral populations.

Competing interests

The authors declare that they have no competing interests.

\section{Authors' contributions}

All authors performed the hatchery and field experiments, drafted the manuscript, and approved the final manuscript. 


\section{Acknowledgements}

We thank M. Ponce, F. Castrence Jr., R. de Guzman, C. Angelito, and A. Rubio III for the research assistance and Drs. R. Dizon, E. Gomez, and H. Yap for the logistical support. The English translation of an article in Japanese cited in the manuscript was kindly provided by Dr. M. Yorifuji. This study was supported by the Department of Science and Technology through the Philippine Council for Agriculture, Aquatic and Natural Resources Research and Development. This is MSI Contribution No. 424

\section{Author details}

${ }^{1}$ The Marine Science Institute, College of Science, University of the Philippines Diliman, Quezon City 1101, Philippines. ${ }^{2}$ Present address: Sesoko Station, Tropical Biosphere Research Center, University of the Ryukyus, Motobu, Okinawa 3422, Japan. ${ }^{3}$ Present address: Marine Ecology Research Centre, School of Environment, Science and Engineering, Southern Cross University, Lismore, New South Wales 2480, Australia.

Received: 6 June 2013 Accepted: 11 November 2013

Published: 19 November 2013

\section{References}

Babcock RC (1985) Growth and mortality in juvenile corals (Goniastrea, Platygyra and Acropora): the first year. Proc 5th Intl Coral Reef Symp 4:355-360

Babcock RC, Baird AH, Piromvaragorn S, Thompson DP, Willis BL (2003) Identification of scleractinian coral recruits from Indo-Pacific reefs. Zool Stud 42:211-226

Baird AH, Babcock RC (2000) Morphological differences among three species of newly settled pocilloporid coral recruits. Coral Reefs 19:179-183

Baria MVB, Guest JR, Edwards AJ, Aliño PM, Heyward AJ, Gomez ED (2010) Caging enhances post-settlement survival of juveniles of the scleractinian coral Acropora tenuis. J Exp Mar Biol Ecol 394:149-153

Birrell CL, McCook LJ, Willis BL, Diaz-Pulido GA (2008) Effects of benthic algae on the replenishment of corals and the implications for the resilience of coral reefs. Oceanogr Mar Biol Annu Rev 46:25-63

Burke L, Reytar K, Spalding M, Perry A (2011) Reefs at risk revisited. World Resources Institute, Washington, D.C.

Clarke PJ, Komatsu T, Bell JD, Lasi F, Oengpepa CP, Leqata J (2003) Combined culture of Trochus niloticus and giant clams (Tridacnidae): benefits for restocking and farming. Aquaculture 215:123-144

Crowe TP, Lee CL, McGuinness KA, Amos MJ, Dangeubun J, Dwiono SAP, Makatipu PC, Manuputty J, N'guyen F, Pakoa K, Tetelepta J (2002) Experimental evaluation of the use of hatchery-reared juveniles to enhance stocks of the topshell Trochus niloticus in Australia, Indonesia and Vanuatu. Aquaculture 206:175-197

Edwards AJ, Gomez ED (2007) Reef restoration concepts and guidelines: making sensible management choices in the face of uncertainty. Coral Reef Targeted Research \& Capacity Building for Management Programme. St. Lucia, p 38

Epstein N, Bak RPM, Rinkevich B (2001) Strategies for gardening denuded coral reef areas: the applicability of using different types of coral material for reef restoration. Restor Ecol 9:432-442

Epstein N, Bak RPM, Rinkevich B (2003) Applying forest restoration principles to coral reef rehabilitation. Aquat Conserv Mar Freshw Ecosyst 13:387-395

Gosselin LA, Qian P-Y (1997) Juvenile mortality in benthic marine invertebrates. Mar Ecol Prog Ser 146:265-282

Graham EM, Baird AH, Connolly SR (2008) Survival dynamics of scleractinian coral larvae and implications for dispersal. Coral Reefs 27:529-539

Heyward AJ, Negri AP (1999) Natural inducers for coral larval metamorphosis. Coral Reefs 18:273-279

Kohler KE, Gill SM (2006) Coral Point Count with Excel extensions (CPCe): a visual basic program for the determination of coral and substrate coverage using random point count methodology. Comput Geosci 32:1259-1269

Klumpp DW, Pulfrich A (1989) Trophic significance of herbivorous macroinvertebrates on the central Great Barrier Reef. Coral Reefs 8:135-144

McCook LJ, Jompa J, Diaz-Pulido G (2001) Competition between corals and algae in coral reefs: a review of evidence and mechanisms. Coral Reefs 19:400-417

Ng CSL, Toh TC, Toh KB, Guest J, Chou LM (2013) Dietary habits of grazers influence their suitability as biological controls of fouling macroalgae in ex situ mariculture. Aquac Res, doi:10.1111/are.12128

Omori M (2005) Success of mass culture of Acropora corals from egg to colony in open water. Coral Reefs 24:563
Omori M, Kubo H, Kajiwara K, Matsumoto H, Watanuki A (2006) Rapid recruitment of corals on top shell snail aquaculture structures. Coral Reefs 25:280

Omori M, Kajiwara K, Matsumoto H, Watanuki A, Kubo H (2007) Why corals recruit successfully in top-shell snail aquaculture structures? Galaxea, Jap Coral Reef Soc 8:83-90

Purcell SW (2000) Association of epilithic algae with sediment distribution on a windward reef in the northern Great Barrier Reer, Australia. Bull Mar Sci 66:199-214

Purcell SW, Cheng HW (2010) Experimental restocking and seasonal visibility of a coral reef gastropod assessed by temporal modeling. Aquat Biol 9:227-238

Rinkevich B (1995) Restoration strategies for coral reefs damaged by recreational activities: the use of sexual and asexual recruits. Restor Ecol 3:241-251

Rinkevich B (2000) Steps towards the evaluation of coral reef restoration by using small branch fragments. Mar Biol 136:807-812

Rinkevich B (2005) Conservation of coral reefs through active restoration measures: recent approaches and last decade progress. Environ Sci Technol 39:4333-4342

Rinkevich B (2006) The coral gardening concept and the use of underwater nurseries: lessons learned from silvics and silviculture. In: Precht WF (ed) Coral reef restoration handbook. CRC Press, Boca Raton, pp 291-301

Sato M (1985) Mortality and growth of juvenile coral Pocillopora damicornis (Linnaeus). Coral Reefs 4:27-33

Secretariat of the Pacific Community (2008) Status report: nearshore and reef fisheries and aquaculture. Secretariat of the Pacific Community. Nouméa New Caledonia, http://www.spc.int/DigitalLibrary/Doc/FAME/Reports/ Anon 08 FisheriesStatusReport.pdf. Accessed 16 Nov 2013

Shafir S, Abady S, Rinkevich B (2009) Improved sustainable maintenance for mid-water coral nursery by the application of an anti-fouling agent. J Exp Mar Biol Ecol 368:124-128

Tamura M (2008) Effects of feeding behavior on juvenile top shell Trochus niloticus on the early developmental stages of coral. Midoriishi 19:37-39

Toh TC, Ng CSL, Guest J, Chou LM (2013) Grazers improve health of coral juveniles in ex situ mariculture. Aquaculture, doi:10.1016/j. aquaculture.2013.08.025

Vicentuan KC, Baria MV, Cabaitan PC, Dizon RM, Villanueva RD, Aliño PM, Gomez ED, Guest JR, Edwards AJ, Heyward AJ (2008) Multi-species spawning of corals in north-western Philippines. Coral Reefs 27:83

Villanueva RD, Yap HT, Montaño MNE (2005) Survivorship of coral juveniles in a fish farm environment. Mar Pollut Bull 51:580-589

Villanueva RD, Edwards AJ, Bell JD (2010) Enhancement of grazing gastropod populations as a coral reef restoration tool: predation effects and related applied implications. Restor Ecol 18:803-809

Wilkinson C (2004) Status of coral reefs of the world: 2004. Australian Institute of Marine Science, Townsville

doi:10.1186/1810-522X-52-44

Cite this article as: Villanueva et al:: Effects of grazing by herbivorous gastropod (Trochus niloticus) on the survivorship of cultured coral spat. Zoological Studies 2013 52:44.

\section{Submit your manuscript to a SpringerOpen ${ }^{\odot}$ journal and benefit from:}

- Convenient online submission

- Rigorous peer review

- Immediate publication on acceptance

- Open access: articles freely available online

- High visibility within the field

- Retaining the copyright to your article

Submit your next manuscript at springeropen.com 